\title{
Factores familiares asociados a la deserción escolar en los niños y niñas mapuche: un estudio de caso*
}

\author{
Family Factors Related to School Dropout in Mapuche Children: A Case Study \\ Fatores familiares associados ao abandono escolar em crianças mapuches: \\ um estudo de caso
}

\begin{abstract}
Óscar Espinoza D., ${ }^{a}$ Dante Castillo G., Luis Eduardo González F., Javier Loyola C. ${ }^{b}$
aPrograma Interdisciplinario de Investigaciones en Educación (PIIE), Centro de Investigación en Educación (CIE), Universidad Ucinf y CPCE, Universidad Diego Portales. Dalmacia 1267, Providencia, Santiago de Chile. Telf.: (56-2) 2096644. Correo electrónico: oespinoza@academia.cl

bPrograma Interdisciplinario de Investigaciones en Educación (PIIE) y Centro de Investigación en Educación (CIE) de la Universidad UCINF.
\end{abstract}

\section{RESUMEN}

El presente artículo se propone como objetivo principal el de evaluar la presencia y el peso específico que tienen, en los desertores escolares de educación básica de origen mapuche pertenecientes a una comuna vulnerable de la ciudad de Santiago, una serie de factores familiares que la literatura especializada ha identificado como variables cruciales a la hora de predecir el abandono. La información sobre la cual se basa este trabajo fue obtenida de una encuesta aplicada a una muestra de niños y niñas que abandonaron la escuela en el ciclo primario. Los resultados muestran que entre niños mapuche y no mapuche existen diferencias significativas en cuanto a ciertas características estructurales, lo cual confirma la necesidad de establecer políticas diferenciadas para prevenir la deserción en este grupo étnico.

Palabras clave: deserción escolar, mapuche, estudiantes vulnerables, factores familiares, educación primaria.

\begin{abstract}
The present article has as its main objective to assess the presence and the specific weight of a set of relevant family factors in primary level mapuche school leavers from a vulnerable area of Santiago. The study is based on quantitative information obtained from a survey applied to a sample of boys and girls living in this area who left school before completing the primary cycle. The results show that mapuche and non-mapuche exhibit major differences with respect to certain structural characteristics, which confirms the need for setting up differentiated policies to prevent dropout among this ethnic group.
\end{abstract}

Key words: school dropout, mapuche, vulnerable students, family factors, elementary education.

\section{RESUMO}

Objetiva-se avaliar a presença e importância específica que tem para estudantes de origem mapuche, desistentes da Educação Básica e pertencentes a uma comunidade vulnerável da cidade de Santiago, uma série de fatores familiares que a literatura especializada identifica como variáveis cruciais na hora de anunciar a desistência. A informação sobre a qual se baseia este trabalho foi obtida a partir de um questionário aplicado a uma mostra de crianças que abandonaram a escola no Ensino Primário. Resultados mostram que entre crianças mapuches e não mapuches há diferenças significativas em termos de certas características estruturais, o que confirma a necessidade estabelecer políticas diferenciadas para prevenir o abandono escolar de este grupo étnico.

Palavras chave: abandono escolar, mapuche, estudantes vulneráveis, fatores familiares, ensino primário.

\footnotetext{
Trabajo derivado del Proyecto Regular de Investigación FONDECYT № 1090730 titulado "Factores que inciden en la deserción escolar y sus implicancias en sectores vulnerables: un estudio de caso", financiado por CONICYT.
} 


\section{INTRODUCCIÓN}

De acuerdo con algunas proyecciones de la Comisión Económica para América Latina y el Caribe (CEPAL, 2005), en países como Argentina, Chile, Colombia, Ecuador, México, Panamá, Perú y Uruguay a lo menos el $95 \%$ de los niños que actualmente tienen menos de cinco años de edad concluirían la educación básica o primaria para el año 2015. Son justamente estos los países que se encuentran más cercanos al logro de la meta trazada por dicho organismo.

En Chile, los estudios recientes sobre deserción escolar en educación básica indican que ésta prácticamente no existe, siendo la cobertura nacional en el nivel primario del $99,5 \%$. No obstante, cuando estas cifras se analizan en mayor detalle, se aprecia que en el quintil más pobre la cobertura disminuye al 98,5\% y que, en el período 1992-2002, sólo un 83,5\% logró egresar de este ciclo en el período de 10 años de la cohorte establecida (Asociación Chilena Pro Naciones Unidas [ACHNU], 2006). Por lo tanto, un $16,5 \%$ del total de la cohorte no terminó, al menos, la educación básica. Este porcentaje no se distribuye aleatoriamente, sino que son los grupos más pobres y excluidos los que en mayor medida sufren este problema (Espíndola y León, 2002).

El vínculo existente entre pobreza, exclusión y deserción escolar obliga a reponer este tema en la agenda de las políticas educativas, considerando que la educación sigue siendo uno de los mecanismos fundamentales de inclusión social de las personas, y por tanto, como derecho humano básico, también es un medio que habilita a los sujetos para el ejercicio amplio de sus derechos. En efecto, quien en la actualidad no cuenta con la educación básica completa está prácticamente excluido de todas las instituciones sociales, culturales, políticas y económicas. Si bien en Chile los porcentajes de deserción son marginales, en el caso de los sectores más vulnerables las cifras muestran que cada año va en aumento el número de niños y jóvenes que se desarrollan por fuera del tipo de vida cívica que se ha definido en las sociedades democráticas.

Indudablemente, esta situación requiere de intervenciones integradas que provean respuestas diversificadas y secuenciadas que favorezcan el reencanto con la educación, la eventual reincorporación al sistema educativo formal o la existencia de ofertas educativas especializadas, en vista del logro de los 12 años de escolaridad con que se ha comprometido el Estado chileno. La posibilidad de éxito de dichas intervenciones radica en la identificación certera de los factores que están llevando a los niños, niñas y jóvenes de sectores vulnerables a abandonar la escuela. Por este motivo, la profundización de las investigaciones relativas al fenómeno de la deserción y retención escolar adquieren una relevancia fundamental, especialmente cuando se trata de grupos sociales que han sido históricamente excluidos y que se caracterizan por presentar indicadores de pobreza superiores al del resto de la población chilena, como es el caso de los grupos indígenas. ${ }^{1}$

En esa perspectiva, y considerada la importancia del problema en cuestión, el presente artículo sintetiza algunos hallazgos del estudio titulado "Factores que inciden en la deserción escolar y sus implicancias en sectores vulnerables: un estudio de caso". La mencionada investigación tiene como objetivo principal analizar los factores que inciden en la deserción escolar en la enseñanza básica en sectores vulnerables, ello a través de la

Según la Encuesta de Caracterización Socioeconómica Nacional (CASEN) de 2009, a este año el 14,8\% de la población no indígena se encontraba en situación de pobreza, mientras que en el caso de la población indígena este porcentaje ascendía a un $19,9 \%$. 
comparación de las trayectorias de desertores y no desertores que presentan condiciones socioeconómicas y educativas homologables. Más específicamente, pretende dar cuenta de los itinerarios educativos y de las trayectorias sociales tanto de niños desertores como de estudiantes pertenecientes a la comuna de Cerro Navia en la ciudad de Santiago, un sector que por sus características puede catalogarse como altamente vulnerable.

Se entiende acá la deserción como un fenómeno de carácter complejo que se construye a partir de dinámicas compuestas, tanto por elementos internos como externos a la escuela, al mismo tiempo que se reconoce que la identificación de estos factores intra y extraescolares es aún insuficiente para los desertores de educación básica, particularmente en Chile donde no se han realizado estudios sistemáticos para este ciclo, así como tampoco investigaciones que diferencien a los niños y niñas en función de su etnia. Es por estas razones que se torna absolutamente necesario describir de un modo más preciso al desertor de educación básica, diferenciándolo del desertor de educación secundaria y considerando entre otras cosas el grupo étnico al cual pertenece.

Así, no sólo es necesario atender a las particularidades de la deserción en educación básica, sino que también evaluar cómo se presenta este fenómeno en el ciclo primario en el caso de los niños y niñas con características étnicas particulares. Esto resulta ser de suma importancia, por cuanto investigaciones de este tipo pueden proveer la base para la elaboración e implementación de políticas diferenciadas con el fin de prevenir el abandono escolar en adolescentes y jóvenes pertenecientes a pueblos originarios, en particular de los mapuche, por tratarse de la etnia de mayor presencia a nivel nacional. ${ }^{2}$

El presente artículo pretende ser un aporte en esta labor, al tener como objetivo central el de evaluar la presencia y el peso específico que en términos comparativos tienen, en los desertores escolares de educación básica de origen mapuche pertenecientes a Cerro Navia -una comuna de Santiago de Chile que se caracteriza por poseer un alto porcentaje de población de esta etnia-, una serie de factores familiares que la literatura especializada sobre deserción ha identificado como variables importantes a la hora de predecir el abandono. De esta forma, el artículo se centra sólo en quienes efectivamente han desertado del sistema escolar y en variables independientes de tipo familiar, estableciendo una comparación entre niños y niñas mapuche y no mapuche en lo relativo a los factores que tienen una mayor incidencia sobre el abandono escolar en cada grupo.

Este trabajo se encuentra dividido en cuatro partes. En la primera sección se hace un repaso de la literatura relevante existente en materia de deserción, con énfasis en los factores extraescolares vinculados a las familias de los niños, niñas y jóvenes. En la segunda sección se expone brevemente la metodología empleada. La tercera parte evalúa qué tan importante es la presencia de algunos de los factores identificados por la literatura especializada en los desertores escolares de origen mapuche, estableciendo una comparación con aquellos que no declaran pertenecer a ningún pueblo originario. Finalmente, se incluye un apartado de conclusiones.

Según datos del CENSO de 2002, del total de habitantes del país que se consideraba perteneciente a grupos étnicos (4,6\%), el 87,3\% declaraba pertenecer a la etnia mapuche. 


\section{FACTORES FAMILIARES ASOCIADOS A LA DESERCIÓN ESCOLAR: ESTADO DEL ARTE}

En este artículo la deserción es comprendida como un proceso de alejamiento y de abandono paulatino de un espacio cotidiano -como es la escuela- que implica también el abandono de ciertos ritos personales y familiares que inciden en el desarrollo de la identidad y la proyección personal de un niño (Comisión Intersectorial de Reinserción Educativa, 2006).

Los factores que originan la deserción del sistema escolar formal se suelen agrupar en dos grandes marcos interpretativos, cuyo énfasis está puesto en variables de índole extraescolar e intraescolar, respectivamente (Espinoza et. al., 2010). En el primero de ellos, que constituye el foco del presente trabajo, se identifica a la situación socioeconómica y al contexto familiar de niños, niñas y jóvenes como las principales causales del abandono escolar. Se mencionan la pobreza y la marginalidad, la búsqueda de trabajo, la disfuncionalidad familiar y las bajas expectativas de la familia con respecto a la educación, entre otros desencadenantes (Pomerantz, Moorman y Litwack, 2007; Castillo, 2003; Croninger y Lee, 2001; Programa de Promoción de la Reforma Educativa de América Latina y el Caribe [PREAL], 2003).

Uno de los resultados más claros y consistentes que ha arrojado la investigación empírica sobre el problema de la deserción escolar es un fuerte vínculo entre el abandono y el estatus socioeconómico, medido este último normalmente a partir del nivel educacional de los padres y del ingreso del grupo familiar (Rumberger, 2001). Una gran cantidad de estudios empíricos han demostrado que los estudiantes que provienen de familias de bajo estatus socioeconómico presentan mayores probabilidades de desertar del sistema escolar que aquellos niños y niñas insertos en familias de estatus socioeconómico medio y alto (Ingrum, 2007; Rumberger y Thomas, 2000; Goldschmidt y Wang, 1999; Janosz et al., 1997; Rumberger, 1995; Haveman, Wolfe y Spaulding, 1991; Bryk y Thum, 1989; Rumberger, 1983)

Otro factor de índole familiar que ha mostrado incidir significativamente sobre la deserción escolar a la luz de la investigación empírica corresponde a la estructura de la familia: los niños y niñas que crecen con ambos padres son más exitosos en la escuela y son menos propensos al abandono escolar que quienes viven en familias monoparentales y en familias reconstituidas (con el/la padre/madre original y un/una padrastro/madrastra o con ninguno de los dos padres originales) (Perreira, Harris y Lee, 2006; Pong y Ju, 2000; Wojtkiewicz, 1993; Sandefur, McLanahan y Wojtkiewicz, 1992; Astone y McLanahan, 1994 y 1991; Krein y Beller, 1988).

En particular, este tipo de familias se asocian con la presencia en los estudiantes de ciertos signos de desencantamiento o falta de compromiso con la escuela, tales como bajas aspiraciones educacionales; bajo rendimiento académico; ausentismo escolar y actitudes negativas hacia trabajar duro en la escuela y entrar a la universidad (Astone y McLanahan, 1991). En el caso específico de las familias monoparentales, una parte significativa de su efecto negativo sobre las tasas de deserción escolar se debe al hecho de que estos grupos familiares en general disponen de menos recursos económicos que las familias que cuentan con la presencia de ambos padres (McLanahan, 1985).

A juicio de Rumberger (2001) resulta absolutamente necesario dar cuenta de los procesos subyacentes a través de los cuales estos factores familiares inciden sobre las tasas de deserción escolar. En este sentido, se ha mencionado que los padres mejor educados 
pueden constituirse como modelos a la vista de sus hijos y que son más propensos a pasar una mayor cantidad de tiempo con ellos, lo que contribuiría a aumentar sus aspiraciones educacionales y su habilidad académica reduciéndose así el riesgo de abandono escolar. Por otro lado, los estudiantes insertos en familias de altos ingresos tendrían una mayor probabilidad de experimentar apoyo y de vivir experiencias educativas gratificantes, al contrario de los niños y niñas provenientes de familias pobres, quienes pueden sentir la presión de desertar de la escuela, de tal modo de ingresar tempranamente al mundo laboral y ayudar económicamente a sus hogares (Rumberger, 1983).

Acorde con lo anterior, Astone y McLanahan (1991) demuestran empíricamente que los niños y niñas que viven en familias monoparentales y reconstituidas se encuentran expuestos a diferentes expectativas de parte de sus padres y a distintos estilos parentales que quienes viven en grupos familiares intactos. Específicamente, en las familias no intactas los padres presentan menores expectativas educacionales y se involucran menos en la educación de sus hijos, vale decir, aquí los niños y niñas reciben menos atención y estímulo parental respecto de sus actividades educativas, todas estas características que se asocian con bajos logros en la escuela y con una mayor probabilidad de abandono escolar. Otras investigaciones empíricas también han mostrado que, en general, los estudiantes que exhiben bajos logros en la escuela y los desertores provienen de familias con padres que tienen bajas expectativas educacionales hacia ellos y que no les proveen de apoyo académico ni monitorean o supervisan sus actividades (Jeynes, 2007; Spera, 2005; Fan y Chen, 2001; Sui-Chu y Willms, 1996; Rumberger, 1995; Rumberger et al., 1990).

El vínculo entre el contexto familiar y la deserción escolar puede visualizarse también a través del concepto de capital cultural (Bourdieu, 1997): las diferencias de capital cultural, cuando se compara el apoyo escolar que reciben los alumnos de parte de las familias al momento de asistir a clases, no sólo se traducen en un buen rendimiento académico, sino que también en un apoyo implícito a la motivación y valoración de la educación, disminuyendo los factores de abandono. En este sentido, algunos estudios muestran que la probabilidad de permanecer en el sistema educacional, junto a los factores asociados al nivel de ingreso familiar, está relacionada en gran medida con la actitud que se genera en los niños y jóvenes a partir del clima educacional que produce la familia (PREAL, 2003).

El fenómeno de la deserción escolar también se desencadena por razones económicas: está unido a la necesidad de ingresar tempranamente al mundo laboral, para de esta forma satisfacer los requerimientos económicos familiares. ${ }^{3}$ Se ha mostrado que el trabajo infantil y adolescente está estrechamente vinculado al abandono, y que su probabilidad crece a medida que aumenta la intensidad del empleo o las horas trabajadas (Perreira, Harris y Lee, 2006; Warren y Cataldi, 2006; Warren y Lee, 2003; McNeal, 1997). Ahora bien, el ingreso prematuro al mercado laboral no sólo está determinado por razones de sobrevivencia o de satisfacción de necesidades básicas, sino que también se relaciona con el poco valor que los jóvenes le atribuyen a su permanencia en los establecimientos escolares. En ese sentido, para un cierto grupo de niños y jóvenes de sectores populares

La respuesta prematura a la demanda de ingreso al trabajo se transforma en sí misma en otro factor de deserción, al tiempo que limita la probabilidad de encontrar trabajos bien remunerados. Los jóvenes de hogares pobres, por lo general, acceden a trabajos precarios y se encuentran expuestos a niveles mínimos de estabilidad laboral. Algunas estimaciones sugieren que en aquellos países en que la deserción escolar tiende a coincidir con haber completado el ciclo primario, el logro de tres años adicionales de educación -hasta completar el primer ciclo de secundaria- redunda en mejoras salariales de entre 30\% y 50\% (PREAL, 2003). 
la promesa de la institución educativa como palanca fundamental de la movilidad social ha perdido fuerza en relación con la exploración temprana de caminos alternativos a la educación formal (CEPAL, 2005; Espíndola y León, 2002; Espinoza, 2000).

\section{METODOLOGÍA}

Para dar cuenta de su objetivo central, en esta investigación se trabajó con una base de datos correspondiente a parte de los desertores escolares de educación básica de Cerro Navia que hicieron abandono de la escuela entre los años 2006 y 2008. El universo o marco muestral se obtuvo mediante la elaboración de un catastro generado a partir de la revisión de los libros de clases en donde se registra la asistencia de los estudiantes. Como desertores escolares fueron considerados aquellos niños y niñas que abandonaron sus estudios en el ciclo primario durante el período 2006-2008 sin haberse matriculado posteriormente en otro establecimiento educacional de Cerro Navia o de otra comuna.

La selección de los casos que componen la muestra de desertores se realizó de forma dirigida o no probabilística. Esto supone una limitación al no poder estimarse la probabilidad de error y el nivel de confianza con el que se hacen las estimaciones. Ahora bien, dada la imposibilidad de conseguir un marco muestral exhaustivo con la limitada y muchas veces inexacta información provista por la comuna (domicilios equivocados, niños y niñas que figuraban como desertores pero que sin embargo habían vuelto a la misma escuela o se habían cambiado a un establecimiento educacional diferente, etc.) y por el Ministerio de Educación, ${ }^{4}$ de todos los casos registrados en el catastro se seleccionó cuidadosamente un subgrupo de ellos que con certeza eran fidedignos: 304 en total. De esta forma, si bien el proceso de selección no fue aleatorio, el procedimiento utilizado buscó aproximarse lo más posible a la consecución de una muestra representativa de la población de referencia. El porcentaje total de población mapuche en la muestra es de $14,5 \%$, equivalente a 44 jóvenes. De ellos, el 36,4\% son mujeres y el 63,6\% son hombres, distribución por sexo muy similar a la que se registra para los no mapuche (Tabla 1).

Tabla 1. Pertenencia a pueblo originario, según sexo de los desertores(as)

\begin{tabular}{|c|c|c|c|c|}
\hline \multicolumn{2}{|c|}{} & \multicolumn{2}{|c|}{ Sexo } & \multirow{2}{*}{ Total } \\
\cline { 3 - 4 } \multicolumn{2}{|c|}{} & Femenino & Masculino & \\
\hline $\begin{array}{c}\text { Pertenencia } \\
\text { a pueblo } \\
\text { originario }\end{array}$ & No mapuche & $32,7 \%(85)$ & $67,3 \%(175)$ & $100 \%(260)$ \\
\cline { 2 - 4 } & Mapuche & $36,4 \%(16)$ & $63,6 \%(28)$ & $100 \%(44)$ \\
\hline \multicolumn{2}{|c|}{ Total } & $33,2 \%(101)$ & $66,8 \%(203)$ & $100 \%(304)$ \\
\hline
\end{tabular}

Fuente: Elaboración propia.

A los casos de desertores incluidos en la muestra mediante el procedimiento recién señalado se les aplicó una encuesta, que en rigor, la mayoría de las veces fue contestada

4 Cabe destacar que el Ministerio de Educación no dispone de un registro de desertores a nivel comunal ni menos a nivel de escuela. 
por algún integrante de su familia (adulto significativo), dada la escasa edad de los menores. Las preguntas contenidas en este cuestionario buscan establecer el perfil de los niños y niñas desertores de educación básica de Cerro Navia y de sus respectivas familias.

Cabe señalar que Cerro Navia es una de las 37 comunas de la ciudad de Santiago de Chile. Se encuentra ubicada en el sector poniente de la capital, y según el último Censo de abril del año 2002 su población estaba constituida por 148.312 habitantes. En comparación con otras comunas del país, Cerro Navia exhibe un alto nivel de pobreza: de acuerdo con los datos de la Encuesta CASEN de 2006, el 17,5\% de su población se hallaba en esta situación, porcentaje superior al registrado tanto a nivel nacional como metropolitano.

\section{RESULTADOS}

\subsection{CARACTERIZACIÓN GENERAL DE LOS ENCUESTADOS}

La encuesta fue contestada en su gran mayoría por mujeres $(80,9 \%)$, lo que preliminarmente revela la ausencia de figuras masculinas relevantes en el entorno de las niñas y niños desertores (Tabla 2). Consecuentemente, se observa una importante proporción de madres $(62,8 \%)$ entre los respondientes, presencia que excede largamente a la de los padres $(5,3 \%)$, los cuales incluso son superados por un punto porcentual por los abuelos (6,3\%). Si se desagregan los resultados por pertenencia a pueblo originario se observa que en el caso de los desertores mapuche el cuestionario fue respondido en un 70,5\% por las madres, porcentaje mayor al que se registra entre los desertores no mapuche $(61,5 \%)$ (Tabla 3).

Tabla 2. Sexo de los encuestados(as)

\begin{tabular}{|c|c|c|}
\hline & Frecuencia & Porcentaje \\
\hline Femenino & 246 & 80,9 \\
\hline Masculino & 58 & 19,1 \\
\hline Total & 304 & 100 \\
\hline
\end{tabular}

Fuente: Elaboración propia.

Tabla 3. Encuestados(as), según pertenencia a pueblo originario

\begin{tabular}{|l|l|c|c|c|}
\hline \multirow{2}{*}{\multicolumn{2}{|c|}{}} & \multicolumn{2}{|c|}{ Pertenencia a pueblo originario } & \multirow{2}{*}{ Total } \\
\cline { 2 - 4 } & No mapuche & Mapuche & \\
\hline \multirow{5}{*}{ Encuestados(as) } & Madre & $61,5 \%(160)$ & $70,5 \%(31)$ & $62,8 \%(191)$ \\
\cline { 2 - 5 } & Padre & $4,6 \%(12)$ & $9,1 \%(4)$ & $5,3 \%(16)$ \\
\cline { 2 - 5 } & Hermana(o) & $4,2 \%(11)$ & $9,1 \%(4)$ & $4,9 \%(15)$ \\
\cline { 2 - 5 } & Abuela(o) & $6,9 \%(18)$ & $2,3 \%(1)$ & $6,3 \%(19)$ \\
\cline { 2 - 5 } & Otro familiar & $11,9 \%(31)$ & $4,5 \%(2)$ & $10,9 \%(33)$ \\
\cline { 2 - 5 } & Otro no familiar & $2,3 \%(6)$ & $0 \%$ & $2 \%(6)$ \\
\cline { 2 - 5 } & El propio desertor(a) & $8,5 \%(22)$ & $4,5 \%(2)$ & $7,9 \%(24)$ \\
\hline Total & $100 \%(260)$ & $100 \%(44)$ & $100 \%(304)$ \\
\hline
\end{tabular}

Fuente: Elaboración propia. 
Esta presencia generalizada de las madres se ve refrendada por el hecho de que son precisamente ellas quienes, la mayor parte de las veces, han actuado como las apoderadas de los jóvenes en la escuela: en el 87,5\% de los casos, porcentaje que entre los mapuche es incluso más alto, llegando al 95,5\% (Tabla 4). Cabe mencionar además, que son pocos los desertores que responden a la encuesta $(7,9 \%)$, debido fundamentalmente a su condición de menores de edad. El porcentaje de jóvenes mapuche que contestó el cuestionario representa sólo el $0,7 \%$ del total de respondientes.

Tabla 4. Apoderados(as), según pertenencia a pueblo originario

\begin{tabular}{|l|l|c|c|c|}
\hline \multicolumn{2}{|c|}{} & \multicolumn{2}{|c|}{ Pertenencia a pueblo originario } & \multirow{2}{*}{ Total } \\
\cline { 2 - 4 } \multicolumn{2}{|c|}{} & No mapuche & M apuche & \\
\hline \multirow{5}{*}{ A poderados } & M adre & $86,2 \%(224)$ & $95,5 \%(42)$ & $87,5 \%(266)$ \\
\cline { 2 - 5 } & Padre & $3,1 \%(8)$ & $2,3 \%(1)$ & $3 \%(9)$ \\
\cline { 2 - 5 } & Hermana(0) & $1,5 \%(4)$ & $0 \%$ & $1,3 \%(4)$ \\
\cline { 2 - 5 } & A buela(o) & $3,1 \%(8)$ & $2,3 \%(1)$ & $3 \%(9)$ \\
\cline { 2 - 5 } & Otro familiar & $3,8 \%(10)$ & $0 \%$ & $3,3 \%(10)$ \\
\cline { 2 - 5 } & Otro no familiar & $2,3 \%(6)$ & $0 \%$ & $2 \%(6)$ \\
\hline Total & $100 \%(260)$ & $100 \%(44)$ & $100 \%(304)$ \\
\hline
\end{tabular}

Fuente: Elaboración propia.

\subsection{DESERTORES ESCOLARES}

La muestra comprende los niños y niñas de Cerro Navia que abandonaron la escuela en el período 2006-2008, siendo este último año el que concentra el porcentaje más alto de deserción $(38,8 \%)$ (Tabla 5). La mayoría de los desertores corresponden a hombres $(66,8 \%)$ (Tabla 6), y la mayor parte de los abandonos se produce en séptimo y octavo básico: ambos niveles reúnen más de la mitad de los casos $(52,6 \%)$. La deserción se produce de este modo con mayor frecuencia en los últimos cursos de la enseñanza básica. En efecto, en los primeros niveles el abandono es bajo y va subiendo progresivamente a medida que se avanza en el ciclo primario, alcanzando su punto máximo de $26,6 \%$ en séptimo básico. Ahora bien, en los mapuche la deserción es mucho más frecuente en el sexto básico: de los 44 jóvenes de esta etnia, el 34,1\% abandonó la escuela en este curso. Entre estos jóvenes, a diferencia de los no mapuche, séptimo y octavo básico no reúnen más de un $50 \%$ de deserción, pero sí lo hacen sexto, quinto y cuarto $(63,6 \%)$ (Tabla 7). Así, en el caso de los mapuche, el abandono escolar parece desencadenarse más tempranamente.

Tabla 5. Año de retiro (Mapuche y no mapuche)

\begin{tabular}{|c|c|c|}
\hline & Frecuencia & Porcentaje \\
\hline 2006 & 85 & 28,0 \\
\hline 2007 & 101 & 33,2 \\
\hline 2008 & 118 & 38,8 \\
\hline Total & 304 & 100 \\
\hline
\end{tabular}

Fuente: Elaboración propia. 
Tabla 6. Sexo de los desertores(as) (Mapuche y no mapuche)

\begin{tabular}{|c|c|c|}
\hline & Frecuencia & Porcentaje \\
\hline Femenino & 101 & 33,2 \\
\hline Masculino & 203 & 66,8 \\
\hline Total & 304 & 100 \\
\hline Total & 304 & 100 \\
\hline
\end{tabular}

Fuente: Elaboración propia.

Tabla 7. Curso al momento del retiro, según pertenencia a pueblo originario

\begin{tabular}{|l|l|c|c|c|}
\hline \multicolumn{2}{|c|}{} & \multicolumn{2}{|c|}{ Pertenencia a pueblo originario } & \multirow{2}{*}{ Total } \\
\cline { 3 - 4 } \multicolumn{2}{|c|}{} & No mapuche & M apuche & \\
\hline \multirow{3}{*}{$\begin{array}{l}\text { Curso al } \\
\text { momento del } \\
\text { retiro }\end{array}$} & Primero & $1,5 \%(4)$ & $0 \%$ & $1,3 \%(4)$ \\
\cline { 2 - 4 } & Segundo & $1,9 \%(5)$ & $2,3 \%(1)$ & $2,0 \%(6)$ \\
\cline { 2 - 4 } & Tercero & $4,6 \%(12)$ & $6,8 \%(3)$ & $4,9 \%(15)$ \\
\cline { 2 - 4 } & Cuarto & $4,2 \%(11)$ & $13,6 \%(6)$ & $5,6 \%(17)$ \\
\cline { 2 - 4 } & Quinto & $15,4 \%(40)$ & $15,9 \%(7)$ & $15,5 \%(47)$ \\
\cline { 2 - 4 } & Sexto & $15,4 \%(40)$ & $34,1 \%(15)$ & $18,1 \%(55)$ \\
\cline { 2 - 4 } & Séptimo & $28,8 \%(75)$ & $13,6 \%(6)$ & $26,6 \%(81)$ \\
\cline { 2 - 4 } & Octavo & $28,1 \%(73)$ & $13,6 \%(6)$ & $26,0 \%(79)$ \\
\hline Total & $100 \%(260)$ & $100 \%(44)$ & $100 \%(304)$ \\
\hline
\end{tabular}

Fuente: Elaboración propia.

\subsection{VARIABLES EXPLICATIVAS DE LA DESERCIÓN ESCOLAR}

Uno de los aspectos más ampliamente relevados por la literatura especializada sobre abandono escolar se refiere a la asociación existente entre altas tasas de deserción y bajo estatus socioeconómico de las familias (Rumberger, 2001). Uno de los indicadores que se utiliza para medir este último es, además del ingreso familiar, el nivel educacional de los padres, en especial de la madre. Aquí los datos confirman la existencia de un vínculo entre el nivel de enseñanza alcanzado y el abandono escolar, ya que el 77,6\% de las madres o apoderados de los desertores no llegó a completar la enseñanza media y, de ellos, un 31,3\% no terminó el ciclo básico y un 4,9\% ni siquiera fue a la escuela. Esta asociación parece ser aún más fuerte entre los mapuche: en las familias de esta etnia el nivel educacional es mucho más bajo si se lo compara con las familias no mapuche. En su caso más del $50 \%$ de las madres no terminó la educación básica $(54,5 \%)$, habiendo un importantísimo $22,7 \%$ que no fue a la escuela. Este último porcentaje equivale a 10 madres, cifra que en términos absolutos supera a la misma correspondiente a los no mapuche, que es de cinco (Tabla 8). 
Tabla 8. Nivel de enseñanza de la madre, según pertenencia a pueblo originario

\begin{tabular}{|l|l|c|c|c|}
\hline \multicolumn{2}{|c|}{} & \multicolumn{2}{|c|}{ Pertenencia a pueblo originario } & \multirow{2}{*}{ Total } \\
\cline { 3 - 4 } \multicolumn{2}{|c|}{} & No mapuche & M apuche & \\
\hline $\begin{array}{l}\text { N ivel de } \\
\text { enseñanza de } \\
\text { la madre }\end{array}$ & No fue a la escuela & $1,9 \%(5)$ & $22,7 \%(10)$ & $4,9 \%(15)$ \\
\cline { 2 - 4 } & Básica incompleta & $31,2 \%(81)$ & $31,8 \%(14)$ & $31,3 \%(95)$ \\
\cline { 2 - 5 } & Básica completa & $21,5 \%(56)$ & $9,1 \%(4)$ & $19,7 \%(60)$ \\
\cline { 2 - 5 } & M edia incompleta & $20,8 \%(54)$ & $27,3 \%(12)$ & $21,7 \%(66)$ \\
\cline { 2 - 5 } & M edia completa & $22,3 \%(58)$ & $6,8 \%(3)$ & $20,1 \%(61)$ \\
\cline { 2 - 5 } & $\begin{array}{l}\text { Superior } \\
\text { incompleta }\end{array}$ & $1,9 \%(5)$ & $2,3 \%(1)$ & $2,0 \%(6)$ \\
\cline { 2 - 5 } & Superior completa & $0,4 \%(1)$ & $0 \%$ & $0,3 \% 1(1)$ \\
\hline Total & $100 \%(260)$ & $100 \%(44)$ & $100 \%(304)$ \\
\hline
\end{tabular}

Fuente: Elaboración propia.

En cuanto al otro indicador de estatus socioeconómico, esto es, el ingreso familiar, el $69,7 \%$ de los encuestados declara que en los últimos tres meses han existido dificultades económicas en sus familias, lo que convierte a los problemas de esta índole en los que concentran la mayor cantidad de menciones. Esta clase de problemas tiene una prevalencia mayor entre las familias mapuche que entre las no mapuche: un altísimo 97,7\% de las primeras declara haberlos tenido últimamente. Junto con esto, porcentualmente casi todos los problemas listados -con la única excepción de malas relaciones entre padres e hijostienen mayor presencia en las familias mapuche, lo que es indicativo de la existencia de un peor clima educacional al interior de estos grupos familiares (Tabla 9).

Tabla 9. Problemas familiares en los últimos tres meses, según pertenencia a pueblo originario (respuestas afirmativas)

\begin{tabular}{|l|c|c|c|}
\hline \multirow{2}{*}{} & \multicolumn{2}{|c|}{ Pertenencia a pueblo originario } & \multirow{2}{*}{ Total } \\
\cline { 2 - 3 } & No mapuche & Mapuche & \\
\hline Falta de comunicación & $54,6 \%$ & $84,1 \%$ & $58,9 \%$ \\
\hline Problemas económicos & $65,0 \%$ & $97,7 \%$ & $69,7 \%$ \\
\hline Problemas con alcohol o drogas & $27,3 \%$ & $54,5 \%$ & $31,3 \%$ \\
\hline Malas relaciones entre padres e hijos & $33,1 \%$ & $9,1 \%$ & $29,6 \%$ \\
\hline $\begin{array}{l}\text { Falta de tiempo para compartir en } \\
\text { familia }\end{array}$ & $43,8 \%$ & $86,4 \%$ & $50,0 \%$ \\
\hline Malas relaciones entre los hermanos & $33,5 \%$ & $59,1 \%$ & $37,2 \%$ \\
\hline Maltrato físico y/o psicológico & $21,9 \%$ & $38,6 \%$ & $24,3 \%$ \\
\hline
\end{tabular}

Fuente: Elaboración propia.

Cabe señalar que en general, a pesar de la existencia de problemas económicos a nivel familiar, estas dificultades no llevan necesariamente a los jóvenes a insertarse de forma temprana en el mundo laboral. Los desertores que actualmente se encuentran trabajando alcanzan sólo el 10,2\%, siendo en su totalidad hombres, ya que todas las mujeres por el momento se encuentran en su casa no desempeñando actividad laboral alguna. Los valores que asumen los estadísticos de asociación para variables categóricas -Chi 
cuadrado, coeficiente de contingencia, Phi y V de Cramer- confirman la independencia existente entre estas dos variables: todos ellos tienen un nivel de significación observado mayor a 0,05 . No obstante, porcentualmente, la cantidad de niños y niñas mapuche que se encuentran trabajando es mucho mayor: representa el 38,6\% del total de jóvenes de esta etnia. En efecto, en términos absolutos el número de jóvenes trabajadores mapuche es mayor que el de no mapuche: 17 versus 14 (Tabla 10). Así, los problemas económicos llevan al grupo de los desertores mapuche a insertarse de manera temprana en el mundo laboral en mayor medida que al de los desertores no mapuche, lo que puede deberse también al hecho de que los problemas económicos parecen ser más importantes entre quienes pertenecen a esta etnia.

Tabla 10. Actividad actual de los desertores(as), según pertenencia a pueblo originario

\begin{tabular}{|c|c|c|c|c|}
\hline & \multicolumn{2}{|c|}{$\begin{array}{c}\text { Pertenencia a pueblo } \\
\text { originario }\end{array}$} & \multirow[t]{2}{*}{ Total } \\
\hline & & No mapuche & Mapuche & \\
\hline \multirow[b]{2}{*}{$\begin{array}{c}\text { Actividad } \\
\text { actual de los } \\
\text { desertores(as) }\end{array}$} & $\begin{array}{c}\text { Sólo } \\
\text { trabajando }\end{array}$ & $5,4 \%(14)$ & $38,6 \%(17)$ & $10,2 \%(31)$ \\
\hline & $\begin{array}{l}\text { Por el } \\
\text { momento } \\
\text { está en la } \\
\text { casa }\end{array}$ & $94,6 \%(246)$ & $61,4 \%(27)$ & $89,8 \%$ (273) \\
\hline \multicolumn{2}{|c|}{ Total } & $100 \%(260)$ & $100 \%(44)$ & $100 \%(304)$ \\
\hline
\end{tabular}

Fuente: Elaboración propia.

Por otra parte, el estatus socioeconómico puede incidir sobre la deserción a través del efecto del nivel educacional de la madre o apoderado sobre las expectativas educacionales y el rendimiento académico de los niños y niñas que estuvieron en la escuela. En cuanto a estas expectativas, y a diferencia de lo que podría esperarse, aquellas listadas en la encuesta reúnen una gran cantidad de respuestas afirmativas (superando en todas las opciones el 50\%), lo que muestra que la mayoría de los jóvenes tienen proyecciones de futuro. En cuanto a las expectativas propiamente educacionales, el 85,9\% de los desertores desearía continuar sus estudios y el 79,6\% obtener un título profesional, lo cual revela que en general está presente en los jóvenes el deseo de terminar el ciclo escolar. Estos porcentajes entre los mapuche corresponden a un 79,5 y 93,2\%, respectivamente, siendo el primero menor y el segundo mayor a los mismos registrados para los niños y niñas no pertenecientes a esta etnia. Por lo general, los mapuche presentan grandes expectativas, que superan la mayoría de las veces a las observadas en el caso de los niños y niñas no mapuche -con excepción de las ya mencionadas, esto es, expectativas para continuar estudios de nivel superior, y las de ganar dinero y mantener a su familia- e incluso con respuestas positivas que muchas veces superan el $90 \%$ (Tabla 11). 
Tabla 11. Expectativas de futuro de los desertores, según pertenencia a pueblo originario (respuestas afirmativas)

\begin{tabular}{|c|c|c|c|}
\hline & No mapuche & Mapuche & \\
\hline Ganar dinero & $77,3 \%$ & $68,2 \%$ & $76,0 \%$ \\
\hline Conseguir un buen trabajo & $82,3 \%$ & $97,7 \%$ & $84,5 \%$ \\
\hline Mantener a su familia & $76,9 \%$ & $68,2 \%$ & $75,7 \%$ \\
\hline Sacar un título & $77,3 \%$ & $93,2 \%$ & $79,6 \%$ \\
\hline Vivir mejor que ahora & $87,3 \%$ & $97,7 \%$ & $88,8 \%$ \\
\hline Ser valorado socialmente & $85,8 \%$ & $100 \%$ & $87,8 \%$ \\
\hline Ser valorado profesionalmente & $81,2 \%$ & $97,7 \%$ & $83,6 \%$ \\
\hline Realizarse como persona & $94,2 \%$ & $97,7 \%$ & $94,7 \%$ \\
\hline Formar una familia & $89,6 \%$ & $95,5 \%$ & $90,5 \%$ \\
\hline Continuar estudios & $86,9 \%$ & $79,5 \%$ & $85,9 \%$ \\
\hline Cambiarse de barrio & $67,7 \%$ & $79,5 \%$ & $69,4 \%$ \\
\hline Poseer casa propia & $83,5 \%$ & $93,2 \%$ & $84,9 \%$ \\
\hline Fuente: Elaboración propia. & & &
\end{tabular}

En cuanto al rendimiento académico, el cual podría verse afectado por la baja escolaridad de la madre o apoderado, los resultados muestran que en términos porcentuales la repitencia es algo mayor entre los desertores mapuche que entre los no mapuche: el 63,6\% de los primeros repitió algún curso, contra el 41,9\% de los segundos (Tabla 12).

Tabla 12. Repitencia entre los desertores, según pertenencia a pueblo originario

\begin{tabular}{|c|c|c|c|c|}
\hline \multicolumn{2}{|c|}{} & \multicolumn{2}{|c|}{$\begin{array}{c}\text { Pertenencia a pueblo } \\
\text { originario }\end{array}$} & \multirow{2}{*}{ Total } \\
\cline { 3 - 4 } \multicolumn{2}{|c|}{} & No mapuche & Mapuche & \\
\hline \multirow{2}{*}{$\begin{array}{c}\text { Repitió algún } \\
\text { curso }\end{array}$} & Sí & $41,9 \%(109)$ & $63,6 \%(28)$ & $45,1 \%(137)$ \\
\cline { 2 - 4 } & No & $58,1 \%(151)$ & $36,4 \%(16)$ & $54,9 \%(167)$ \\
\hline \multicolumn{2}{|c|}{ Total } & $100 \%(260)$ & $100 \%(44)$ & $100 \%(304)$ \\
\hline
\end{tabular}

Fuente: Elaboración propia.

Otro factor predictor de la deserción identificado por la literatura corresponde a la estructura de la familia. En relación con este aspecto se verifica que menos de la mitad de los desertores considerados $(43,8 \%)$ vive con su padre; por el contrario, en la gran mayoría de los casos $(88,2 \%)$ los menores viven con su madre. También se advierte una presencia importante de abuelos (45,7\%, mayor al 43,8\% de los padres) y tíos $(34,9 \%)$ en las familias de los desertores, así como también de hermanos (77,3\%), aunque esto último puede explicarse por otras razones. De este modo, los resultados confirman que los desertores en su mayoría se encuentran insertos en familias monoparentales. Al desagregar los resultados por etnia llama la atención que la totalidad de los desertores mapuche vive con su madre y su(s) hermano(as), y que sólo un 29,5\% lo hace con su padre, porcentaje 
que es bastante menor al registrado para los desertores no mapuche. Este último porcentaje indica, asimismo, el número de familias con presencia de ambos padres existente dentro de los desertores mapuche (Tabla 13).

Tabla 13. Personas que viven con los desertores(as), según pertenencia a pueblo originario (respuestas afirmativas)

\begin{tabular}{|c|c|c|c|}
\hline & $\begin{array}{c}\text { Pertenencia } \\
\text { a pueblo } \\
\text { originario }\end{array}$ & Total & \\
\hline & No mapuche & Mapuche & \\
\hline Madre & $86,2 \%$ & $100 \%$ & $88,2 \%$ \\
\hline Padre & $46,2 \%$ & $29,5 \%$ & $43,8 \%$ \\
\hline Abuela(o) & $47,7 \%$ & $34,1 \%$ & $45,7 \%$ \\
\hline Tía(o) & $34,2 \%$ & $38,6 \%$ & $34,9 \%$ \\
\hline Hermana(o) & $73,5 \%$ & $100 \%$ & $77,3 \%$ \\
\hline Ser valorado profesionalmente & $81,2 \%$ & $97,7 \%$ & $83,6 \%$ \\
\hline Realizarse como persona & $94,2 \%$ & $97,7 \%$ & $94,7 \%$ \\
\hline Formar una familia & $89,6 \%$ & $95,5 \%$ & $90,5 \%$ \\
\hline Continuar estudios & $86,9 \%$ & $79,5 \%$ & $85,9 \%$ \\
\hline Cambiarse de barrio & $67,7 \%$ & $79,5 \%$ & $69,4 \%$ \\
\hline Poseer casa propia & $83,5 \%$ & $93,2 \%$ & $84,9 \%$ \\
\hline
\end{tabular}

Fuente: Elaboración propia.

Junto con la importante presencia de la figura materna, así como también de otros integrantes en desmedro del padre, se advierte también que las familias de los desertores escolares considerados se caracterizan por ser bastante numerosas: el promedio general de personas que viven en el hogar es de 6,51. Este promedio es menor para los mapuche que para los no mapuche: 5,91 versus 6,61 (Tabla 14).

Tabla 14. Número de personas que viven en la casa, según pertenencia a pueblo originario

\begin{tabular}{|c|c|c|c|c|c|c|}
\hline $\begin{array}{c}\text { Pertenencia a } \\
\text { pueblo originario }\end{array}$ & Media & $\mathrm{N}$ & $\begin{array}{c}\text { Desviación } \\
\text { Estándar }\end{array}$ & Mínimo & Máximo & Rango \\
\hline No mapuche & 6,61 & 260 & 2,583 & 3 & 15 & 12 \\
\hline Mapuche & 5,91 & 44 & 2,055 & 4 & 13 & 9 \\
\hline Total & 6,51 & 304 & 2,522 & 3 & 15 & 12 \\
\hline
\end{tabular}

Fuente: Elaboración propia.

\section{CONCLUSIONES}

Los resultados obtenidos permiten establecer algunas importantes diferencias entre el grupo de desertores mapuche y no mapuche pertenecientes a Cerro Navia. Se observa, en 
primer lugar, que la deserción se desencadena de forma más temprana entre los primeros. En los mapuche el abandono se concentra en los niveles intermedios del ciclo primario, mientras que en los no mapuche este fenómeno se presenta más frecuentemente en los dos últimos cursos de la enseñanza primaria.

En cuanto a las variables familiares que la literatura especializada asocia con el fenómeno de la deserción, el estatus socioeconómico aparece con más fuerza en los desertores de origen mapuche de la comuna. Así, las familias de esta etnia presentan, en términos comparativos, niveles educacionales consistentemente más bajos, así como también una mayor presencia de problemas económicos. En su caso, el bajo nivel de enseñanza alcanzado por la madre o apoderado no incide sobre las expectativas de futuro de los niños y niñas mapuche, las cuales son bastante altas, pero sí parece expresar una incapacidad de parte de estas figuras para traspasar el tipo de capital cultural relevante para el sistema escolar (Bourdieu, 2003). Esto se puede observar en un pobre rendimiento académico, que se plasma en niveles de repitencia mayores en comparación con los registrados para los desertores no mapuche, cuestión que finalmente puede conducir al abandono de la escuela.

En lo referente a los problemas económicos, los datos muestran que éstos llevan a los jóvenes desertores mapuche en mayor medida que a los no mapuche a insertarse tempranamente en el mundo laboral, lo que puede resultar incompatible con la permanencia dentro del sistema. Además, junto con las dificultades de esta índole hay otros problemas de mayor presencia entre los mapuche (por ejemplo, los problemas con el alcohol y drogas y de maltrato físico y/o psicológico) que pueden ser indicativos de la existencia de un clima educacional desmejorado al interior de estas familias que puede favorecer la deserción (PREAL, 2003).

El hecho de que los desertores mapuche y sus familias presenten un estatus socioeconómico menor muestra que son este tipo de características de orden estructural las que marcan la diferencia entre este grupo y los no mapuche. Esto no hace sino confirmar la situación general de mayor vulnerabilidad que presenta esta población, incluso en sectores ya de por sí caracterizados por sus altos niveles de pobreza y marginalidad como es el caso de la comuna de Cerro Navia. Ciertamente esta situación exige la elaboración de medidas diferenciadas para prevenir el abandono escolar de los niños y niñas mapuche, los cuales no deben considerarse aisladamente, sino dentro del marco de una política general dirigida hacia este grupo étnico. La definición de las características que deben asumir estas medidas requiere la realización de estudios más comprehensivos que apunten a identificar de forma exhaustiva las variables específicas que inciden sobre la deserción en los mapuche. En el presente trabajo se intentó dar un primer paso en este sentido, considerada la escasa investigación que se ha realizado sobre el tema.

\section{REFERENCIAS BIBLIOGRÁFICAS}

Asociación Chilena Pro Naciones Unidas (2006). La deserción escolar en Chile. ¿Prioridad en la agenda educativa? Santiago de Chile: Foro Nacional Educación de Calidad para Todos, UNESCO. Consultado el 7 de julio de 2009, del sitio Web de la Red Latinoamericana sobre Inclusión Educativa en Ciudades: http://www.redligare.org/IMG/pdf/desercion_escolar_chile_ flamey.pdf

Astone, N.M. y Mclanahan, S.S. (1991). Family structure, parental practices and high school completion. American Sociological Review, vol. 56, n. 3, 309-320. 
(1994). Family structure, residential mobility, and school dropout: A research note. Demography, vol. 31, n. 4, 575-584.

Bourdieu, P. (2003). Los herederos: los estudiantes y la cultura. Buenos Aires: Siglo XXI. (1997). Capital cultural, escuela y espacio social. México: Siglo XXI.

Bryk, A.S. y Thum, M. (1989). The effects of high school organization on dropping out: An exploratory investigation. American Educational Research Journal, vol. 26, n. 3, 353-383.

Castillo, D. (2003). Desertores de la educación básica. Reflexiones e interrogantes a partir de la práctica. Revista Latinoamericana de Innovaciones Educativas, n. 37, 69-90.

Comisión Económica para América Latina y el Caribe. (2005). Objetivos de desarrollo del milenio: Una mirada desde América Latina y el Caribe, Santiago de Chile: Naciones Unidas. Consultado el 21 de junio de 2009, del sitio Web de CEPAL: http://www.eclac.org/publicaciones/xml/1/21541/lcg2331e.pdf

Comisión Intersectorial de Reinserción Educativa. (2006). Programa intersectorial de reescolarización: Construyendo alternativas educativas para niños, niñas y adolescentes en situación de vulnerabilidad, Santiago de Chile: Foro Nacional Educación de Calidad para Todos, UNESCO. Consultado el 7 de julio de 2009, del sitio Web de la Red Latinoamericana sobre Inclusión Educativa en Ciudades: http://www.redligare.org/IMG/pdf/desercion_escolar_chile_flamey.pdf

Croninger, R.G. y Lee, V.E. (2001). Social capital and dropping out of high school: Benefits to at-risk students of teachers' support and guidance. Teachers College Record, n. 103, 548-581.

Espíndola, E. y León, A. (2002). La deserción escolar en América Latina: Un tema prioritario para la agenda regional. Revista Iberoamericana de Educación, n. 30, 39-62.

Espinoza, O., Castillo, D., Gonzalez, L.E. \& Loyola, J. (2010). Discusión teórica en torno a los determinantes de la deserción escolar. Documento de Trabajo CIE N³. Santiago, Universidad Ucinf. En http://www.ucinf.cl/files/CIE_doc_discusion_teorica

Espinoza, V. (2000). Ciudadanía y juventud. Análisis de los perfiles de oferta y demanda de las políticas sociales ante la realidad juvenil. Santiago de Chile: USACH, Instituto de Estudios Avanzados.

Fan, X. y Chen, M. (2001). Parental involvement and students' academic achievement: A metaanalysis. Educational Psychology Review, n. 13, 1-22.

Goldschmidt, P. y Wang, J. (1999). When can schools affect dropout behaviour? A longitudinal multilevel analysis. American Educational Research Journal, vol. 36, n. 4, 715-738.

Haveman, R., Wolfe, B. y Spaulding, J. (1991). Childhood events and circumstances influencing high school completion. Demography, vol. 28, n. 1, 133-157.

Ingrum, A. (2007). High school dropout determinants: The effect of poverty and learning disabilities. The Park Place Economist, vol. 14, 73-79.

Instituto Nacional de Estadísticas (2003). CENSO 2002. Síntesis de Resultados. La Nación. Consultado el 21 de junio de 2009, del sitio Web del INE: http://www.ine.cl/cd2002/sintesiscensal.pdf

Jeynes, W.H. (2007). The relationship between parental involvement and urban secondary school student academic achievement - A meta-analysis. Urban Education, n. 42, 82-110.

Janosz, M., Leblanc, M., Boulerice, B. y Tremblay, R. (1997). Disentangling the weight of school dropout predictors: A test on two longitudinal samples. Journal of Youth and Adolescence, vol. 26, n. 6, 733-762.

Krein, S.F. y Beller, A.H. (1988). Educational attainment of children from single-parent families: Differences by exposure, gender and race. Demography, vol. 25, n. 2, 221-234.

Mclanahan, S. (1985). Family structure and the reproduction of poverty. The American Journal of Sociology, vol. 90, n. 4, 873-901.

Mcneal, R.B. (1997). Are Students Being Pulled Out of High School? The Effect of Adolescent Employment on Dropping Out. Sociology of Education, vol. 70, n. 3, 206-220. 
Ministerio de Planificación (2009). CASEN 2009. Encuesta de Caracterización Socioeconómica Nacional, Santiago de Chile: MIDEPLAN. Consultado el 21 de junio de 2009, del sitio Web del MIDEPLAN: http://www.mideplan.gob.cl/casen2009/RESULTADOS_CASEN_2009.pdf

Perreira, K.M., Harris, K.M. y Lee, D. (2006). Making it in America: High school completion by immigrant and native youth. Demography, $n .43,511-536$.

Pomerantz, E.M., Moorman, E.A. y Litwack, S.D. (2007). The how, whom, and why of parents' involvement in children's academic lives: More is not always better. Review of Educational Research, $n$. 77, 373-410.

Pong, S.-L. y JU, D.B. (2000). The effects of change in family structure and income on dropping out of middle and high school. Journal of Family Issues, n. 21, 147-169.

Programa de Promoción de la Reforma Educativa en América Latina y el Caribe (2003). Deserción escolar: Un problema urgente que hay que abordar. Formas y Reformas de la Educación, Serie Políticas, vol. 5, n. 14, 1-4. Consultado el 25 de junio de 2009, de: http://www.yorku. ca/hdrnet/images/uploaded/Desercion_Escolar.pdf

Rumberger, R.W. (2001). Why students dropout of school and what can be done. Trabajo presentado en la Conferencia Dropouts in America: How severe is the problem? What do we know about intervention and prevention? Harvard University.

(1995). Dropping out of middle school: A multilevel analysis of students and schools. American Educational Research Journal, vol. 32, n. 3, 583-625.

(1983). Dropping out of high school: The influence of race, sex, and family background. American Educational Research Journal, vol. 20, n. 2, 199-220.

Rumberger, R.W. y Thomas, S.L. (2000). The distribution of dropout and turnover rates among urban and suburban high schools. Sociology of Education, vol. 73, n. 1, 39-67.

Rumberger, R.W., Ghatak, R., Poulos, G., Ritter, P.L. y Dornbusch, S.M. (1990). Family influences on dropout behaviour in one California high school. Sociology of Education, vol. 63, n. 4, 283-299.

Sandefur, G.D., Mclanahan, S. y Wojtkiewicz, R.A. (1992). The effects of parental marital status during adolescence on high school graduation. Social Forces, vol. 71, n. 1, 103-121.

Spera, C. (2005). A review of the relationship among parenting practices, parenting styles, and adolescent school achievement. Educational Psychological Review, vol. 17, 120-146.

Sui-Chu, E.H. y Willms, J.D. (1996). Effects of parental involvement on eighth-grade achievement. Sociology of Education, vol. 69, n. 2, 126-141.

Warren, J.R. y Cataldi, E.F. (2006). A historical perspective on high school students' paid employment and its association with high school dropout. Sociological Forum, n. 21, 113-143.

Warren, J.R. y Lee, J.C. (2003). The impact of adolescent employment on high school dropout: Differences by individual and labor-market characteristics. Social Science Research, 32, 98-128.

Wojtkiewicz, R.A. (1993). Simplicity and complexity in the effects of parental structure on high school graduation. Demography, vol. 30, n. 4, 701-717. 